\title{
Short paired-end reads trump long single- end reads for expression analysis
}

\author{
Adam H. Freedman, John M. Gaspar and Timothy B. Sackton *i]
}

\author{
* Correspondence: tsackton@g. \\ harvard.edu \\ Informatics Group, Harvard \\ University, Cambridge, MA 02138, \\ USA
}

\begin{abstract}
Background: Typical experimental design advice for expression analyses using RNAseq generally assumes that single-end reads provide robust gene-level expression estimates in a cost-effective manner, and that the additional benefits obtained from paired-end sequencing are not worth the additional cost. However, in many cases (e.g., with Illumina NextSeq and NovaSeq instruments), shorter paired-end reads and longer single-end reads can be generated for the same cost, and it is not obvious which strategy should be preferred. Using publicly available data, we test whether short-paired end reads can achieve more robust expression estimates and differential expression results than single-end reads of approximately the same total number of sequenced bases.

Results: At both the transcript and gene levels, $2 \times 40$ paired-end reads unequivocally provide expression estimates that are more highly correlated with $2 \times$ 125 than $1 \times 75$ reads; in nearly all cases, those correlations are also greater than for $1 \times 125$, despite the greater total number of sequenced bases for the latter. Across an array of metrics, differential expression tests based upon $2 \times 40$ consistently outperform those using $1 \times 75$.

Conclusion: Researchers seeking a cost-effective approach for gene-level expression analysis should prefer short paired-end reads over a longer single-end strategy. Short paired-end reads will also give reasonably robust expression estimates and differential expression results at the isoform level.
\end{abstract}

Keywords: RNA-seq, Short read sequencing, Differential expression

\section{Background}

For over a decade, RNA-seq has empowered gene expression analysis. This has led to fundamental advances in our understanding of diverse phenomena, including the evolution of gene regulation across species [1], alternative splicing [2, 3], relative importance of cis vs -trans regulation $[4,5]$, genetic underpinnings of heritable disease [6, 7], and the genetic architecture of phenotypes in natural populations $[8,9]$. While the cost of sequencing has steadily decreased, making expression analyses feasible even on modest research budgets, maximizing cost efficiency remains high priority. Cost effectiveness is particularly important as studies scale up to hundreds or even thousands of

(c) The Author(s). 2020 Open Access This article is licensed under a Creative Commons Attribution 4.0 International License, which permits use, sharing, adaptation, distribution and reproduction in any medium or format, as long as you give appropriate credit to the original author(s) and the source, provide a link to the Creative Commons licence, and indicate if changes were made. The images or other third party material in this article are included in the article's Creative Commons licence, unless indicated otherwise in a credit line to the material. If material is not included in the article's Creative Commons licence and your intended use is not permitted by statutory regulation or exceeds the permitted use, you will need to obtain permission directly from the copyright holder. To view a copy of this licence, visit http//creativecommons.org/licenses/by/4.0/. The Creative Commons Public Domain Dedication waiver (http://creativecommons.org/publicdomain/zero/1.0/) applies to the data made available in this article, unless otherwise stated in a credit line to the data. 
samples. Maximizing performance given a particular study design has already received much attention, with investigations of optimal strategies for quality trimming [10], the effects of quality trimming on expression estimates and differential expression testing [11], optimal sequencing depth [12, 13], importance of biological replicates [12, 14, 15], and the relative performance of expression and differential expression tools and pipelines [16-20]. Less attention has focused on how to improve performance given a fixed number of biological replicates and total sequenced bases.

While the typical length of sequenced reads has increased as costs have decreased, when an annotated genome is available it remains standard procedure to sequence single end reads to estimate gene expression, typically around 75 base pairs. Yet, this strategy does not leverage information provided by the full length of the library fragments, particularly the greater specificity in read mapping (or pseudo-alignment) that would be provided by sequencing from both ends of a fragment. Given a fixed cost per base for the Illumina NextSeq, HiSeq X Ten, and NovaSeq instruments, paired-end $75 \mathrm{bp}$ reads would be twice as expensive as single-end 75 bp sequencing, assuming an equivalent number of sequenced fragments. However, a sizeable fraction of the long-range information provided by paired-end sequencing should still be retained by sequencing shorter paired-end reads. In this study, we evaluate whether that intuition is correct, by assessing whether expression estimates and differential expression results obtained with $2 \times 40$ paired end sequencing are more consistent with $2 \times 125$ paired end sequencing than either $1 \times 75$ or $1 \times 125$ single-end sequencing. Our particular focus is on $1 \times 75$, as the cost of $1 \times 75$ and $2 \times 40$ are the same per read when sequencing using the Illumina NextSeq ( 75 cycle kit), a common instrument for RNA-seq studies. Given the fixed cost per base for the HiSeq X Ten and NovaSeq instruments, there are analogous choices to make between single-end vs. paired-end sequencing. For example, for the 100 bp NovaSeq kit, a sequencing strategy trade-off exists between sequencing $2 \times 50$ vs. $1 \times 100$ reads. We conduct these analyses with publicly available Illumina sequence data across 12 different SRA (Additional file 1: Table S1). accessions encompassing diverse experimental designs and multiple model organisms with high quality annotated genomes.

\section{Results}

Our approach to evaluating the performance of short paired-end reads vs. longer single-end ones is to compare expression estimates and differential expression results derived from these strategies to a truth set. Because we cannot know the "truth", we assume that its closest approximation are results obtained from long, paired-end reads. Thus, we define our gold standard as the results obtained from $2 \times 125$ paired-end reads. We then trim these data down to two data sets with read lengths of 40 and 75 base pairs, and calculate Spearman's rank correlations between transcripts-per-million (TPM) estimates based upon $2 \times 40,1 \times 75$ and $1 \times 125$ with the $2 \times 125$ gold standard. We present these correlations in two different ways. First, we examine the distributions of those correlation coefficients across alternative sequencing strategies. Second, we plot the correlations obtained using $2 \times 40$ over those obtained with $1 \times 75$ and $1 \times 125$.

Kallisto transcript-level TPM estimates made with $2 \times 40$ are always more highly correlated with those made with the $2 \times 125$ gold standard than those made with data trimmed to $1 \times 75$ reads (Fig. $1 \mathrm{a}, \mathrm{c}$ ), and perform better than estimates made with data 


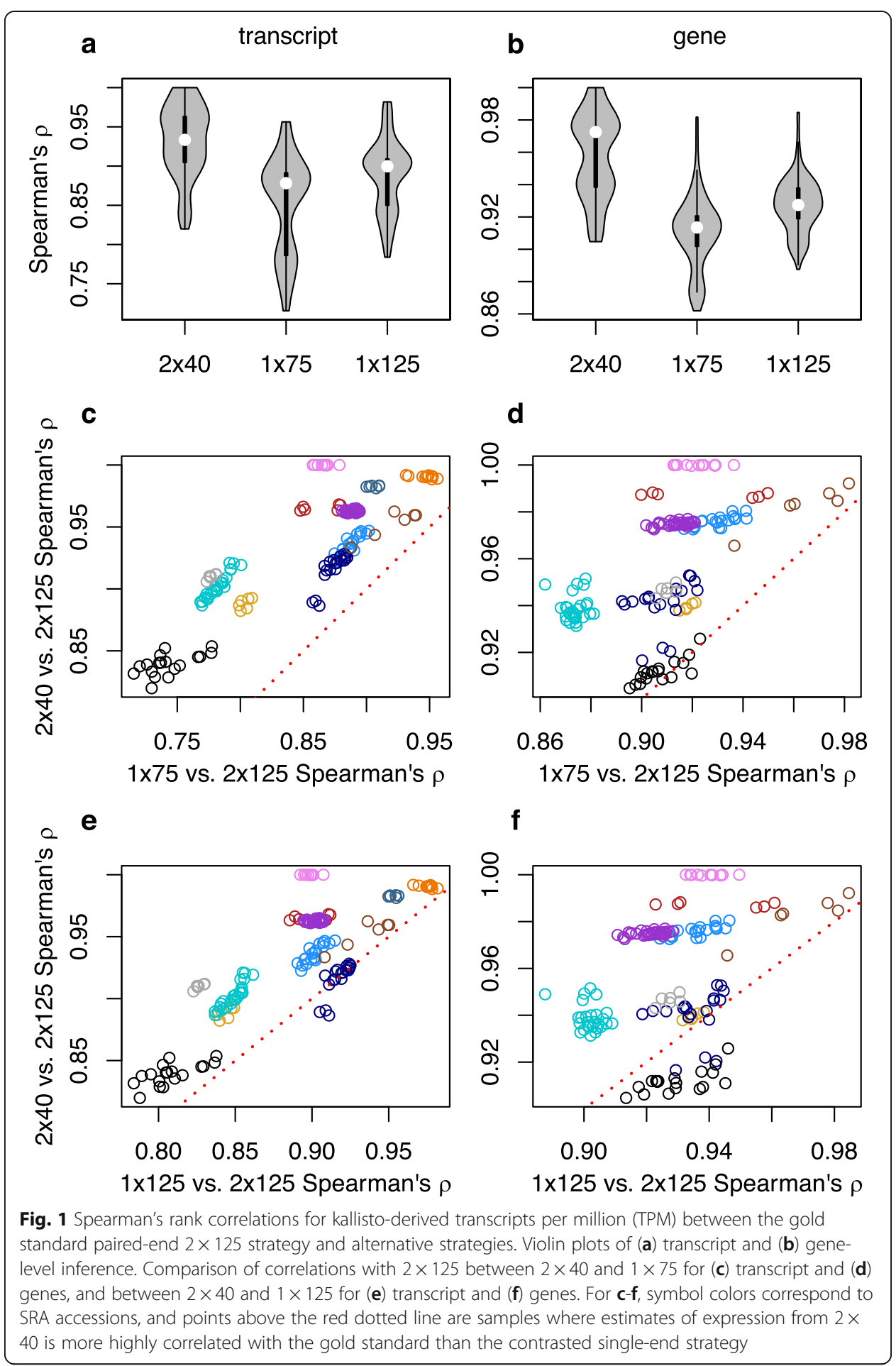

trimmed to $1 \times 125$ for all but a few samples from one SRA accession (Fig. 1a, e). A similar performance advantage for the $2 \times 40$ strategy compared to the $1 \times 75$ strategy is observed at the gene level (Fig. 1b, d), although there is one accession where $1 \times 125$ performs better than $2 \times 40$ (Fig. 1f).

RSEM-based TPM estimates show an overall pattern generally consistent with that observed with kallisto (Fig. 2). A handful of samples for which $2 \times 40$ performed less 




well than both $1 \times 75$ and $1 \times 125$ had low bowtie 2 alignment rates to the reference transcripts (for the $2 \times 125$ libraries, overall alignment rates of 37.1-51.0\%), suggesting that library quality issues were responsible, but raising a broader issue concerning whether variation in alignment rates between strategies could explain the relative performance of $2 \times 40$ to $1 \times 75$ and $1 \times 125$. 
Kallisto does not conduct formal sequence alignment, so for each sample-strategy combination we summed the kallisto transcript-level counts as a proxy for the number of informative reads used in estimating expression. The summed kallisto counts for $2 \times$ 40 have a weak correlation with performance (with respect to TPM) relative to $1 \times 75$ the transcript level, and a moderate correlation at the gene level (Fig. 3a,b); the relative differences in counts between $2 \times 40$ and $1 \times 75$ appear uncorrelated with the relative performance of $2 \times 40$ and $1 \times 75$ (Fig. 3c,d). A similar pattern is observed with respect to $1 \times 125$ (Additional file 1: Figure S1). For RSEM-derived expression estimates, we found no impact of the percent of reads uniquely aligning on the relative performance of $2 \times 40$, but at the gene level found that relative differences in overall alignment rate had some effect on the robustness of TPM estimates of $2 \times 40$ relative to both $1 \times 75$ and $1 \times 125$ (Additional file 1: Figure S2, S3). For both kallisto and RSEM, while there are no obvious taxonomic effects there are clear experiment-specific effects, manifested as differences in relative $2 \times 40$ performance among accessions derived from the same species. Similar to the above results based upon expression correlations, looking across all accessions, for most samples root-mean-square error of expression estimates based upon $1 \times 75$ and $1 \times 125$ was greater than for $2 \times 40$ (Fig. 4).

We next evaluated the extent to which differences in performance with respect to estimating expression levels translated into downstream effects on tests of differential expression. Within each SRA accession and for each pair of conditions and for each sequencing strategy, we conducted Wald tests with, sleuth, limma-voom, and DESeq2.

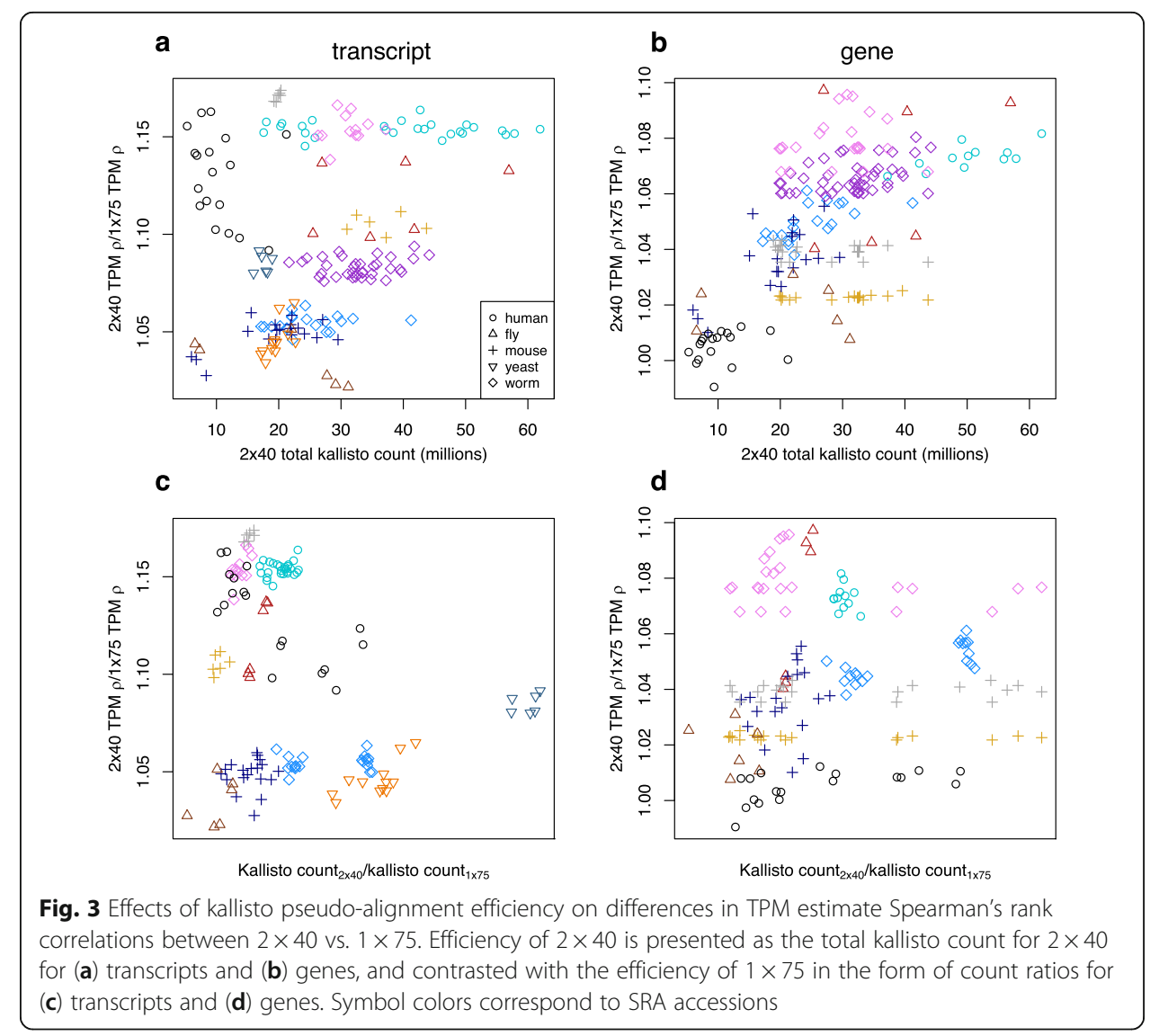




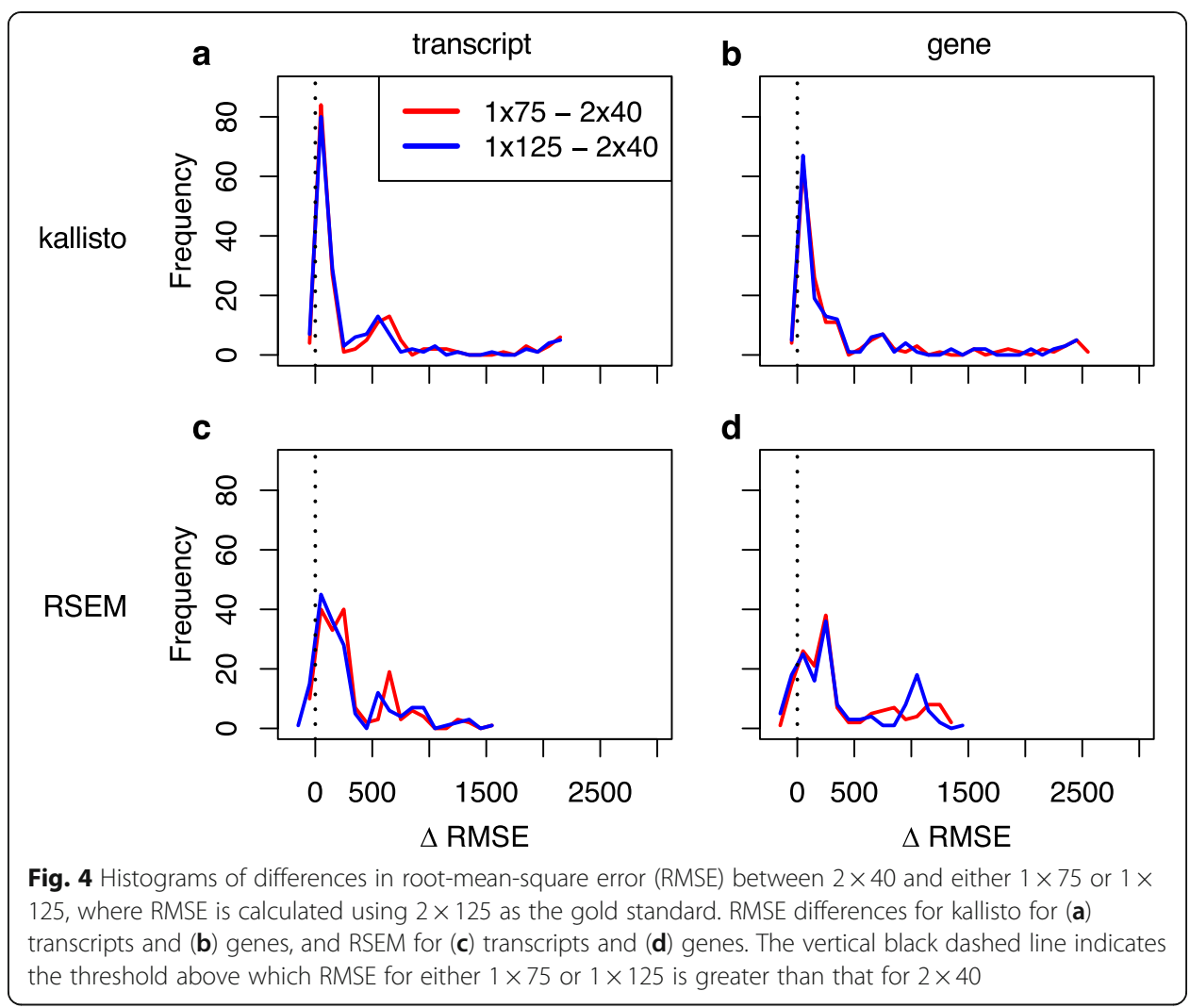

Similar to our evaluation of expression estimate robustness, we defined "true" differential expression signals as those recovered with $2 \times 125$, given a false discovery rate (FDR) of 0.01 . We then calculated seven performance metrics on a per-accession basis for each differential expression method -sequencing strategy combination.

Sequencing strategy clearly impacts downstream differential expression analyses. Looking across all three differential expression methods, at both the transcript and gene level, for the majority of accessions false negative rates are lower for $2 \times 40$ compared to $1 \times 75$ (Fig. 5; Additional file 1: Figure S4, S5; Additional file 1: Table S2). The empirical false discovery rate-that is, for a strategy being evaluated, the proportion of putative differentially expressed features with a Benjamini-Hochberg adjusted $p$-value below our chosen FDR threshold of 0.01 for which the adjusted p-value is $>0.01$ with $2 \times 125$-was also lower for $2 \times 40$ compared to both $1 \times 75$ and $\times 125$, although it was consistently higher than 0.01 , the threshold used to classify Wald tests as significant, including the $2 \times 125$ analyses with which we defined true positives and negatives (Fig. 5; Additional file 1: Figure S4, S5; Additional file 1: Table S2). FDR control was poorer at the transcript than the gene level, and, as previously reported, DESeq2 did not control FDR as well as limma-voom and sleuth [18]. At both the transcript and gene level, AUC is greater for $2 \times 40$ than $1 \times 75$ for all but a handful of method-accession combinations (Fig. 5, Additional file 1: Figure S4, S5; Additional file 1: Table S2). Additional metrics indicate a similar overall performance advantage of $2 \times 40$ compared to $1 \times 75$ (Additional file 1: Table S2). For all metrics, the short paired-end strategy outperforms $1 \times 125$ for the majority of method-accession combinations, although the magnitude of 


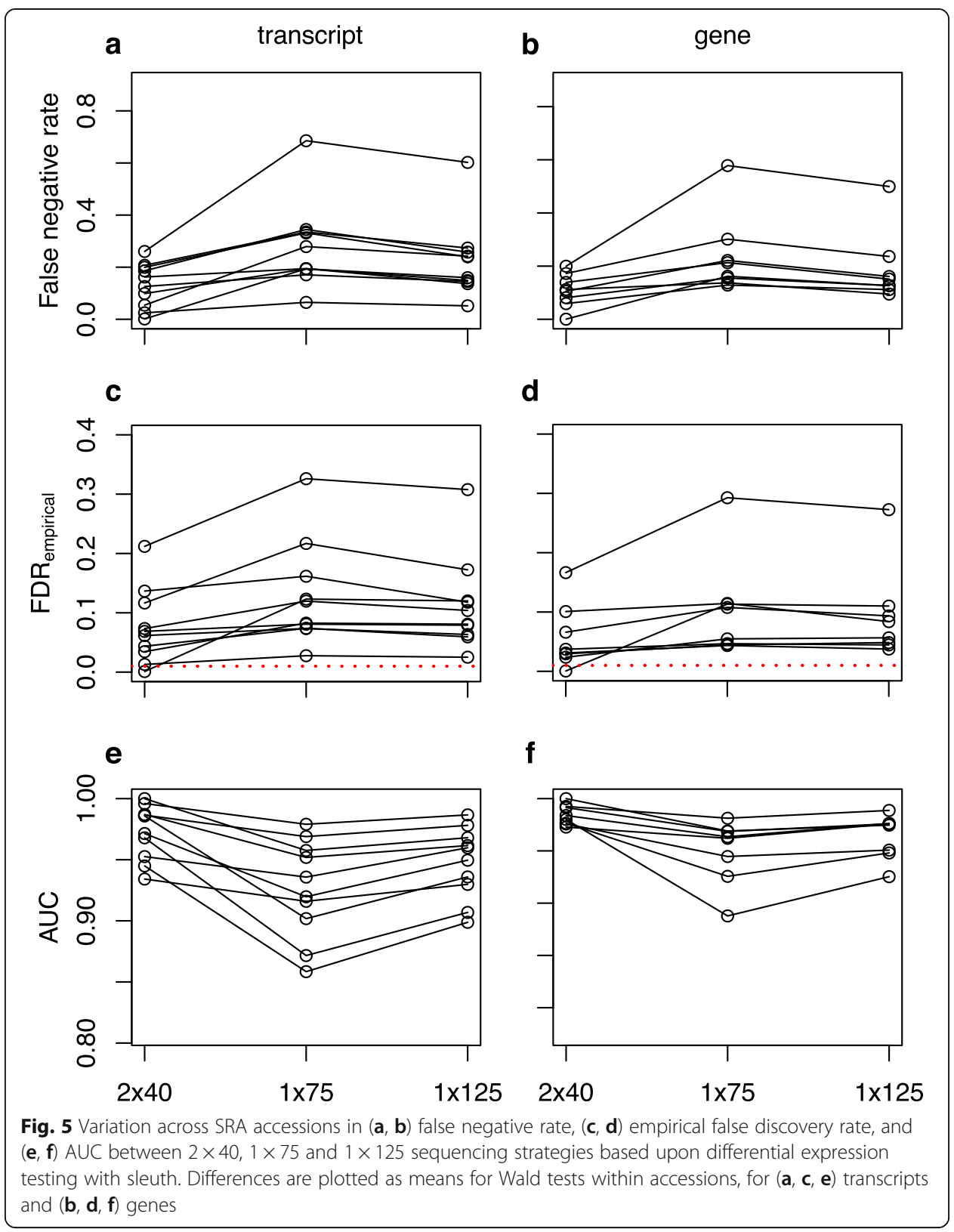

performance advantage of $2 \times 40$ compared to $1 \times 125$ is smaller than when compared with $1 \times 75$ smaller (Fig. 5, Additional file 1: Figure S4,S5; Additional file 1: Table S2).

\section{Discussion}

In the past, the use of single-end sequencing for bulk RNA-seq studies has been motivated by cost concerns, the belief that gene-level analyses of expression are sufficient to investigate many if not all phenomena of interest, and the assumption that moderatesized single-end reads are the best way to balance cost and performance. The continued decline in sequencing costs has been offset by a research-driven need for greater biological replication, and the growth in scale and complexity of study designs, such that cost concerns continue to be important for many researchers. In addition, while genelevel expression estimates continue to drive many research programs, interest in 
isoform-level patterns such as isoform switching or allele-specific expression, continues to grow. Thus, affordable sequencing strategies that generate robust estimates of isoform expression, and enable isoform-level tests of differential expression, would be valuable.

Across multiple datasets and species, and using two different methods to estimate expression levels, we found that short paired-end reads yield both gene and isoform-level expression estimates closer to those obtained with long paired-end reads than moderate sized single-end reads. Notably, this performance advantage occurs when approximately the same number of bases are sequenced in both strategies. This means that, for the same overall cost, short paired-end reads will yield more robust expression estimates. Furthermore, short paired-end reads also outperformed longer single-end reads even when single-end strategy included more than a third more sequenced bases that the short paired-end strategy. While our original hypothesis motivating this study was that improved sequence alignment-both in terms of overall rate and precision-would lead to superior performance of short paired-end reads, surprisingly alignment statistics showed, at best, a weak effect on relative performance, suggesting that additional factors may play a role. Finally, using three different methods to test for DE, we found that the improvement in expression estimation translates to better performance in DE testing, which the most common primary research objective in bulk RNA-seq studies. Thus, across a wide array of bulk RNA-seq applications in in settings where sequencing costs represent a substantial constraint, the use of short paired-end reads has the potential to improve both candidate gene discovery and the detection of biologically meaningful isoform-level variation.

\section{Conclusions}

When designing a gene expression study using bulk RNA-seq, given a fixed budget, more robust expression estimates and differential expression test results can be obtained from short paired-end reads compared to longer single-end reads. In other words, the extra information provided by reads pairs during the mapping or pseudoalignment phase outweighs any potential penalty of short reads. In almost all cases, short paired-end reads trump single-end reads that sequence approximately the same number of bases. Short paired-end reads provide an additional benefit in that they can identify a large fraction of differentially expressed isoforms, for the cost of gene-level analysis with longer single-end reads. From a practical perspective, neither NextSeq nor NovaSeq sequencing kits distinguish between single and paired-end libraries. In the experience of support staff at the Bauer sequencing core facility at Harvard, one can get $92 \mathrm{bp}$ from the NextSeq 75 bp kit, such that $2 \times 40$ is a viable sequencing design. Alternatively, the smallest NovaSeq kit is for 100 cycles, which can be used to sequence paired 50 bp reads, allowing one to leverage that instrument's much smaller cost per sequenced base. Overall, our findings suggest that for a given number of sequencing cycles, the most efficient design for RNA-seq expression analysis is sequencing shorter paired reads, rather than longer single-end reads.

\section{Methods}

Datasets

From NCBI's Short Read Archive, we downloaded paired-end RNA-seq reads from 12 different studies (Additional file 1: Table S1), representing model organisms with well- 
annotated reference genomes: mouse (Mus musculus), human (Homo sapiens), yeast (Saccharomyces cerevisiae), fly (Drosophila melanogaster), and worm (Caenorhabditis elegans). In selecting data sets, we required minimum pre-processing read lengths to be $\geq 125 \mathrm{bp}$, and experimental designs to contain $\geq 2$ experimental conditions.

\section{Short-read processing}

We removed adapters and trimmed reads with NGmerge [21]. For each sample, we trimmed reads to 125,75 , and 40 bp using awk. For downstream analyses, we used $2 \times$ $125,2 \times 40,1 \times 125$, and $1 \times 75$ trimmed data sets. Single-end data sets were comprised of the first read from the original paired reads. Reads were trimmed from the distal ends, such that trimming mimicked how sequencing to different lengths would be carried out on an Illumina sequencer.

\section{Expression analyses}

For each sample-sequencing strategy combination, we estimated transcript-level expression with kallisto [22]. We designated abundance estimates derived from $2 \times 125$ as our gold standard against which to benchmark all other strategies. We used Spearman's rank correlations to quantify the degree of concordance between alternative sequencing strategies and the gold standard in terms of transcript per million (TPM). We performed similar analyses at the gene level by aggregating transcript-level TPMs using the R package Tximport [19]. For yeast, which has no alternative splicing, analyses were only conducted at the transcript level. As an additional estimate of the correspondence between alternative sequencing strategies with $2 \times 125$ we also calculated root-meansquare error (RMSE).

Because a large proportion of RNA-seq applications involve tests of differential expression (DE), we compared differential expression test results between the gold standard $2 \times 125$ and alternative strategies. Specifically, for each experiment we conducted all possible pairwise Wald tests of transcript-level DE with sleuth [18] version 0.30.0, and used $p$-value aggregation (https://pachterlab.github.io/sleuth_walkthroughs/pval_agg/ analysis.html) for gene-level analyses. To compare performance of alternative strategies relative to the gold standard, we calculated false negative and false positive rates, defining significant tests as those with Benjamini-Hochberg adjusted $p$-values $\leq 0.01$.

In order to evaluate whether our findings were pipeline-specific, perhaps related to particular features of tools such as kallisto that rely on pseudo-alignment, we also performed expression analyses with an alternative pipeline, involving estimation of expression levels with RSEM [23] version 1.2.29 based upon alignments with bowtie2 version 2.3.2, [24]. When SRA metadata enabled us to determine that libraries were stranded, we included the relevant RSEM command line arguments for restricting bowtie2 alignments to the correct read pair configuration. Using these expression estimates, we conducted differential expression tests with two different methods: limma-voom [25] version 3.38.3 and DESeq2 [26] version 1.22.2. For limma-voom, we only conducted tests where, for each gene or transcript, there were $\geq 2$ samples with counts per million $(\mathrm{CPM}) \geq 1$. For limma-voom, we employed TMM normalization, and the voomWithQualityWeights option. Sleuth and limma-voom have previously been demonstrated to be top performers that correctly control FDR [22], and while DESeq2 has been shown 
to not control FDR as well as these two methods [22], we included it because it is a commonly used differential expression method. For all differential expression analyses, we calculated performance metrics for Wald tests where, at the specified false discovery rate (FDR) threshold of 0.01 , there were $\geq 50$ significantly differentially expressed features in all sequencing strategies. Metrics were calculated relative to $2 \times 125$, i.e. significant and non-significant results obtained with $2 \times 125$ were treated as true positives and negatives, respectively. Those metrics were the empirical false discovery rate, false positive rate, false negative rate, sensitivity, specificity, precision, and area under the curve (AUC) based upon receiver-operator statistics. These metrics were calculated with python scripts available at our associated github repository (see below). As the multiple-test adjusted $p$-values are the standard metric with which to determine whether particular transcripts or genes are differentially expressed, for AUC calculations we used these values as the response variable relative to the "true" classification of differences derived from tests based upon the $2 \times 125$ data. All reference genomes and gtf-formatted annotations against which to estimate expression were downloaded from Ensembl, and transcripts for kallisto-based analyses were extracted using the extract-reference-transcripts utility in RSEM.

\title{
Supplementary information
}

Supplementary information accompanies this paper at https://doi.org/10.1186/s12859-020-3484-z.

Additional file 1. Supporting Online Material containing additional supplemental figures and tables.

\begin{abstract}
Abbreviations
AUC: Area under curve; DE: Differential expression; FDR: False discovery rate; RMSE: Root-mean-square error; SRA: NCBI short read archive; TMM: Trimmed mean of M values; TPM: Transcript per million

\section{Acknowledgments}

The computations in this paper were run on the Odyssey cluster supported by the FAS Division of Science, Research Computing Group at Harvard University. We thank Claire Reardon, director of the Bauer Core at Harvard University for helpful discussions during the study design phase of our study, and for feedback on preliminary results. We thank Emma White at the Bauer Core for details concerning available sequencing read lengths and kit sizes for Illumina instruments. We also thank members from the Harvard FAS Informatics Group for feedback on preliminary results produced by this study. This work was conducted on the traditional territory of the Wampanoag and Massachusett peoples.
\end{abstract}

Authors' contributions

TBS and AHF designed the experiments, AHF and JMG analyzed the data, and AHF wrote the paper with TBS. The authors read and approved the final manuscript.

\section{Funding}

This study was funded by the Harvard University Division of Science. Harvard had no role in determining the nature or scope of our study.

\section{Availability of data and materials}

The datasets analyzed during the current study and which support the conclusions of this article are publicly available Illumina short read data available on NCBI's Short Read Archive (https://www.ncbi.nlm.nih.gov/sra). Specific accessions are listed in Additional file 1: Table S1. Command lines for executing NGmerge, kallisto, RSEM, sleuth, and limma-voom analyses are available on GitHub at https://github.com/harvardinformatics/rnaseq_readlength_assessment.

Ethics approval and consent to participate

Not applicable.

Consent for publication

Not applicable.

Competing interests

The authors declare that they have no competing interests. 
Received: 10 October 2019 Accepted: 7 April 2020

Published online: 19 April 2020

\section{References}

1. Brawand D, Soumillon M, Necsulea A, Julien P, Csárdi G, Harrigan P, et al. The evolution of gene expression levels in mammalian organs. Nature. 2011;478(7369):343-8.

2. Barbosa-Morais NL, Irimia M, Pan Q, Xiong HY, Gueroussov S, Lee LI, et al. The evolutionary landscape of alternative splicing in vertebrate species. Science. 2012;338(6114):1587-93.

3. Frumkin I, Yofe I, Bar-Ziv R, Gurvich Y, Lu Y-Y, Voichek Y, et al. Evolution of intron splicing towards optimized gene expression is based on various Cis- and trans-molecular mechanisms. PLoS Biol. 2019;17(8):e3000423.

4. Crowley JJ, Zhabotynsky V, Sun W, Huang S, Pakatci IK, Kim Y, et al. Analyses of allele-specific gene expression in highly divergent mouse crosses identifies pervasive allelic imbalance. Nat Genet. 2015;47(4):353-60.

5. Verta J-P, Jones FC. Predominance of cis-regulatory changes in parallel expression divergence of sticklebacks. de Meaux J, Tautz D, editors. eLife. 2019;8:e43785.

6. Castel SE, Cervera A, Mohammadi P, Aguet F, Reverter F, Wolman A, et al. Modified penetrance of coding variants by cis-regulatory variation contributes to disease risk. Nat Genet. 2018;50(9):1327-34.

7. Gandal MJ, Zhang P, Hadjimichael E, Walker RL, Chen C, Liu S, et al. Transcriptome-wide isoform-level dysregulation in ASD, schizophrenia, and bipolar disorder. Science. 2018;362(6420):eaat8127.

8. Campbell-Staton SC, Cheviron ZA, Rochette N, Catchen J, Losos JB, Edwards SV. Winter storms drive rapid phenotypic regulatory, and genomic shifts in the green anole lizard. Science. 2017;357(6350):495-8.

9. Mallarino R, Henegar C, Mirasierra M, Manceau M, Schradin C, Vallejo M, et al. Developmental mechanisms of stripe patterns in rodents. Nature. 2016;539(7630):518-23.

10. MacManes MD. On the optimal trimming of high-throughput mRNA sequence data. Front Genet. 2014;5 [cited 2019 Sep 16]. Available from: https://www.ncbi.nlm.nih.gov/pmc/articles/PMC3908319/.

11. Williams CR, Baccarella A, Parrish JZ, Kim CC. Trimming of sequence reads alters RNA-Seq gene expression estimates BMC Bioinformatics. 2016:17:103.

12. Tarazona S, García-Alcalde F, Dopazo J, Ferrer A, Conesa A. Differential expression in RNA-seq: a matter of depth. Genome Res. 2011:21(12):2213-23.

13. Liu Y, Zhou J, White KP. RNA-seq differential expression studies: more sequence or more replication? Bioinforma Oxf Engl. 2014;30(3):301-4.

14. Todd EV, Black MA, Gemmell NJ. The power and promise of RNA-seq in ecology and evolution. Mol Ecol. 2016;25(6) 1224-41.

15. Robles JA, Qureshi SE, Stephen SJ, Wilson SR, Burden CJ, Taylor JM. Efficient experimental design and analysis strategies for the detection of differential expression using RNA-sequencing. BMC Genomics. 2012;13(1):484.

16. Fonseca NA, Marioni J, Brazma A. RNA-Seq gene profiling - a systematic empirical comparison. PLoS One. 2014;9(9): e107026.

17. Kanitz A, Gypas F, Gruber AJ, Gruber AR, Martin G, Zavolan M. Comparative assessment of methods for the computational inference of transcript isoform abundance from RNA-seq data. Genome Biol. 2015;16:150.

18. Pimentel $\mathrm{H}$, Bray NL, Puente $S$, Melsted P, Pachter L. Differential analysis of RNA-seq incorporating quantification uncertainty. Nat Methods. 2017;14(7):687-90.

19. Soneson C, Love MI, Robinson MD. Differential analyses for RNA-seq: transcript-level estimates improve gene-level inferences. F1000Research. 2015:4:1521.

20. Soneson C, Delorenzi M. A comparison of methods for differential expression analysis of RNA-seq data. BMC Bioinformatics. 2013;14(1):91.

21. Gaspar JM. NGmerge: merging paired-end reads via novel empirically-derived models of sequencing errors. BMC Bioinformatics. 2018;19(1):536

22. Bray NL, Pimentel H, Melsted P, Pachter L. Near-optimal probabilistic RNA-seq quantification. Nat Biotechnol. 2016;34(5): 525-7.

23. Li B, Dewey CN. RSEM: accurate transcript quantification from RNA-Seq data with or without a reference genome. BMC Bioinformatics. 2011;12:323.

24. Langmead B, Salzberg SL. Fast gapped-read alignment with bowtie 2. Nat Methods. 2012;9(4):357-9.

25. Law CW, Chen Y, Shi W, Smyth GK. voom: precision weights unlock linear model analysis tools for RNA-seq read counts. Genome Biol. 2014;15:R29.

26. Love MI, Huber W, Anders S. Moderated estimation of fold change and dispersion for RNA-seq data with DESeq2. Genome Biol. 2014;15(12):550

\section{Publisher's Note}

Springer Nature remains neutral with regard to jurisdictional claims in published maps and institutional affiliations. 